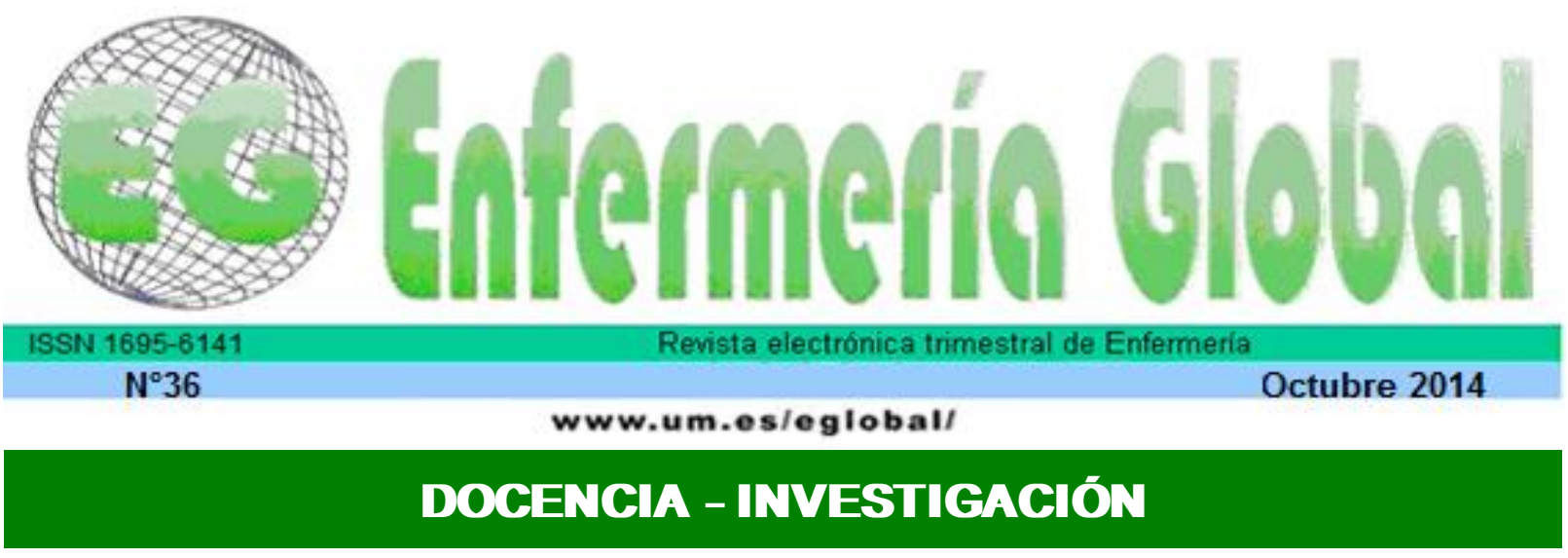

\title{
Rehabilitar en psiquiatría ¿quema al personal de enfermería?
}

Rehabilitate in psychiatry, Does it burn nursing staff out?

\section{*Leal Docampo, Purificación *De Pablo Jarque, Isabel}

\author{
*Enfermera del Servicio de Rehabilitación Psiquiátrica O Rebullón. Xerencia de Xestión Integrada de \\ Vigo. Pontevedra. E-mail: purificacion.leal.docampo@sergas.es
}

\begin{abstract}
Palabras clave: Desgaste profesional (burnout); personal de enfermería de rehabilitación psiquiátrica; CDPE-A; variables sociodemográficas; personalidad resistente
\end{abstract}

Keywords: Wear professional (burnout); nurses psychiatric rehabilitation; CDPE-A; socio-demographic variables; hardiness.

\section{RESUMEN}

El objetivo de este estudio fue analizar el desgaste profesional en el personal de enfermería dedicado a la rehabilitación psiquiátrica hospitalaria. Para ello se tuvieron en cuenta tanto las tres dimensiones específicas de este síndrome, es decir, agotamiento emocional, despersonalización y falta de realización profesional, además de otras variables organizacionales como posibles fuentes de estrés y las consecuencias para la salud física, psicológica, sociofamiliar y laboral; así como el papel desempeñado en el desarrollo de este proceso de algunas variables sociodemográficas y de personalidad. Estos datos, que recoge el cuestionario CDPE-A de Moreno Jiménez, sobre una muestra de 42 profesionales de enfermería del Hospital de Rehabilitación Psiquiátrica O Rebullón de Vigo (España), fueron analizados a través de estadística descriptiva e inferencial.

Los resultados evidencian una baja presencia del síndrome de desgaste profesional en esta población de estudio, con poca relevancia de las variables sociodemográficas y laborales estudiadas, encontrando efectos significativos a nivel bivariado sólo en función de edad e hijos, y sin efectos principales a nivel multivariante; sin embargo, nos muestran unos niveles altos en todas las dimensiones de la escala de la personalidad resistente, así como fuertes mecanismos de afrontamiento tanto directo como en la percepción de apoyo social, lo que posibilita interpretar que estas variables pudieran estar interviniendo como factores preventivos ante el desarrollo del síndrome y por tanto podrían servir de indicadores de salud laboral y calidad asistencial

\section{ABSTRACT}

The aim of this study was to analyze the burnout on nurses dedicated to inpatient psychiatric rehabilitation. This took into account the three specific dimensions of the syndrome (emotional exhaustion, depersonalization and lack of professional accomplishment), organizational type variables as potential sources of stress, the consequences for the physical, psychological, social and family labor and the role played in the development of this process of socio-demographic variables and personality. These data, which includes the questionnaire CDPE-A Moreno Jiménez, on a sample of 42 nurses 
Psychiatric Rehabilitation Hospital O Rebullón of Vigo (Spain), were analyzed by descriptive and inferential statistics.

The results indicate a low presence of burnout syndrome in the study population. Low relevance of sociodemographic and work variables with significant bivariate level effects only in terms of age and children, and no main effects multivariate level. However we show high levels in all dimensions of the scale of hardiness, and strong coping mechanisms both directly and in the perception of social support, which makes it possible to interpret these variables may be intervening as protective factors against development of the syndrome and thus may serve as indicators of occupational health and quality of care.

\section{INTRODUCCIÓN}

El concepto de estrés ya aparece introducido en el ámbito de la salud en el año 1926 por Hans Seleyec como una respuesta del organismo ante cualquier estímulo estresor o situación estresante ${ }^{(1)}$. Mientras que la primera aproximación al proceso de desgaste profesional, entendido como una fase avanzada de estrés laboral, la encontramos en 1974, cuando el psiquiatra Herbert Freudenberger definió el "síndrome del quemado o burnout" como fallar, agotarse, o llegar a desgastarse debido a un exceso de fuerza, demandas excesivas de energía o de recursos ${ }^{(2)}$. Paralelamente, Maslach y Jackons se centraron en el estrés emocional que surge de la relación interpersonal con los clientes y, en los años 80, desarrollaron el cuestionario "Maslach Burnout Inventory" (MBI) como instrumento para evaluar el burnout, considerándolo como un proceso gradual de agotamiento emocional, progresivo cinismo y despersonalización y disminución de la eficacia o realización profesional ${ }^{(3)}$. El agotamiento emocional haría referencia a los sentimientos de no poder dar más de sí a nivel emocional; la despersonalización a la aparición de respuestas de distancia negativa como las actitudes cínicas hacia otras personas que normalmente son las destinatarias del servicio o cuidado; y la baja realización profesional correspondería a una autoevaluación negativa de competencia y logro en el trabajo. La etiología y desarrollo de este proceso también podría explicarse, de manera general, por la presencia de altas demandas de la tarea y escasos recursos o capacidades individuales. Y en el caso de personas dedicadas a profesiones de ayuda podría decirse que las demandas interpersonales llegan a consumir tanto capital emocional que para evitar el contacto con la fuente originaria del malestar se adopta la despersonalización como estrategia de afrontamiento, lo que llevaría en una última instancia a una reducida realización profesional ${ }^{(4)}$.

Aunque el desgaste profesional, como una fase avanzada de estrés crónico producido tras el desequilibrio de las expectativas entre el ámbito profesional y la realidad del trabajo diario ${ }^{(5)}$, no puede restringirse a profesionales de los servicios sociales y sanitarios, sin embargo, es en estos campos donde se ha encontrado más evidencia, especialmente en el personal que atiende pacientes críticos y terminales ${ }^{(6)}$. A su vez, dentro de las profesiones sanitarias, el personal de enfermería ha sido identificado como un grupo de profesionales con alto riesgo de sufrir agotamiento y estrés, tal como lo demuestran diversos estudios realizados en todo el mundo ${ }^{(7,8)}$. Esto es debido a la gran cantidad de estresores inherentes a su trabajo, que afrontan diariamente, y por su relación directa con las personas enfermas, caracterizada por continuas e intensas demandas físicas y emocionales durante largos periodos de tiempo, y donde, constantemente, se enfrenta a pobres prognosis, al sufrimiento humano, la invalidez y la muerte ${ }^{(9)}$. $\mathrm{O}$, como sucede en el área de salud mental, donde el manejo del paciente psiquiátrico es altamente estresante si se carece de ciertas habilidades ${ }^{(10)}$. 
Además de la sobrecarga de trabajo, podrían añadirse otros factores laborales de tipo organizativo-administrativo que aumentarían la vulnerabilidad ante el desgaste como serían el insuficiente reconocimiento y apoyo desde la dirección, la falta de compañerismo, así como el conflicto y ambigüedad del rol por límites poco claros en la responsabilidad de funciones y obligaciones, la falta de comunicación o la limitada participación en la toma de decisiones. Existen algunas investigaciones que corroboran que los niveles altos de cohesión suelen reducir el estrés ocupacional, mientras que el apoyo de la dirección y el compañerismo protegen de las obligaciones adicionales que crean los pacientes ${ }^{(11)}$.

La literatura confirma la relación inversa entre el desgaste profesional y la satisfacción laboral ${ }^{(12)}$, y a la vez demuestra la falta de consenso a la hora de elaborar un perfil de riesgo dado la disparidad de resultados encontrados en las distintas investigaciones llevadas a tal efecto ${ }^{(13)}$. Si bien se acostumbra a tener en cuenta la relación de algunas variables socidemográficas como facilitadoras o inhibidoras del desarrollo del síndrome, algunos autores han llegado a señalar la escasa o nula relación entre las características sociodemográficas y el síndrome de burnout. Por otro lado existen cada vez más trabajos que ponen de manifiesto que factores individuales como la personalidad resistente o alguna de sus dimensiones pudieran actuar de forma directa e indirecta reduciendo la posibilidad de experimentar este desgaste.

La fortaleza o personalidad resistente fue un constructo propuesto por Kobasa en 1979. Se compone de tres variables: compromiso, control y desafío o reto. Mientras las dos primeras se asociarían específicamente con la protección del desgaste, el reto se relacionaría negativamente con la falta de realización ${ }^{(7)}$ al desarrollar una función de resistencia ante la ocurrencia de un estimulo estresante, lo que supondría que antes de ser evitado o evaluado como una amenaza a la propia seguridad sería entendido como una oportunidad y un incentivo para el crecimiento personal (14). Resultados de varios estudios en esta línea confirman la relación entre personalidad resistente y la salud o bienestar psíquico ${ }^{(15)}$.

La necesidad de estudiar el estrés laboral como uno de los precursores del deterioro de la salud mental deriva, y sobre todo en España, desde el hincapié hecho con la aparición de la nueva Ley en Prevención de Riesgos Laborales (Ley 31/1995, de 8 de noviembre, BOE 10-11-1995) "al reconocer la organización y ordenación del trabajo como condiciones de trabajo susceptibles de producir riesgos laborales" (art.4, apartado 7.d). Las razones que recomiendan prestar atención a los problemas de salud mental van desde considerarlos responsables de absentismo y bajas laborales, al abandono de la profesión, así como la repercusión que tiene el bienestar y la salud laboral sobre la eficacia de una organización dada la retroalimentación existente entre calidad de vida, estado de salud física y mental y la organización. En las conclusiones de la Conferencia Ministerial de la OMS para la Salud Mental en Helsinki, en 2005, la OMS establecía la adopción de medidas para la prevención del absentismo y la enfermedad mental derivadas de situaciones de estrés laboral ${ }^{(16)}$.

Teniendo en cuenta que el desgaste profesional supone una experiencia compuesta de cogniciones, emociones y actitudes negativas hacía el trabajo, hacia las personas con las que se trabaja y hacia el propio rol profesional. Que a esto le acompañan consecuencias disfuncionales a nivel fisiológico, psicológico y conductual, que van a tener repercusiones nocivas para las personas y la organización, y que suelen aparecer por la utilización de estrategias de afrontamiento inadecuadas ante el estrés laboral generado por las relaciones con los clientes y la organización ${ }^{(2)}$. Y sin obviar 
que algunas investigaciones también confirman una alta prevalencia de burnout y conducta suicida ${ }^{(17)}$; no cabe dudar que el estudio de este síndrome se convierte en una obligada necesidad social orientada a mejorar la salud y la calidad de vida de las personas.

Partiendo de la hipótesis de que el personal de enfermería es una población susceptible a desarrollar el síndrome de desgaste profesional, y que este es un indicador incuestionable de pérdida de salud, que repercute tanto en la satisfacción laboral como en la calidad de servicio de la organización, se desarrolla este estudio con el objetivo de comprobar los niveles de desgaste presentes en las y los profesionales de enfermería que desarrollan su trabajo en un hospital de rehabilitación psiquiátrica, así como las consecuencias y algunos factores organizacionales, sociodemográficos y personales que pudieran estar implicadas en este proceso, y con el fin de promover las intervenciones enfocadas a su prevención dentro del contexto de la Salud Laboral y la Prevención de Riesgos Laborales.

\section{MATERIAL Y MÉTODO}

Diseño: Se trata de un estudio descriptivo de corte transversal llevado a cabo en un hospital de rehabilitación psiquiátrica de Vigo.

Muestra y procedimiento: La población objeto de estudio fue el total del personal de enfermería del Hospital $O$ Rebullón $(\mathrm{N}=69)$, distribuido en cuatro unidades. El procedimiento, tras autorización de la dirección de enfermería del CHUVI, fue el reparto de sobres cerrados dirigidos nominalmente a cada profesional conteniendo el cuestionario con un carta de presentación, agradecimiento e información sobre el estudio a realizar y la confidencialidad de los datos, además de un sobre para guardar el cuestionario una vez cumplimentado y dirigido a la unidad de docencia para asegurar el anonimato de las personas encuestadas. La participación fue voluntaria, siendo la tasa de respuesta durante todo el mes de mayo de 2012 (periodo calendarizado para ello) del 65\%; si bien, del total de los 45 cuestionarios recogidos y analizados, en una nueva revisión se consideraron 42 válidos, lo que redujo la muestra aquí analizada a un $61 \%$ de la población total.

Instrumentos de medición: Se utilizó la versión abreviada CDPE-A del Cuestionario de Desgaste Profesional de Enfermería (CDPE) que es un cuestionario construido para la evaluación específica del desgaste profesional (burnout) en el colectivo de enfermería, que ya fue utilizado para este fin en otros estudios y en esta ocasión fue cedido y autorizado por Moreno Jiménez, autor junto con Garrosa y González de dicho cuestionario. El CDPE, con 174 ítems, se fundamenta en un modelo teórico transaccional e interactivo, que considera la aparición del desgaste profesional de enfermería como resultado del contexto laboral de esta profesión, de las características individuales y del tipo de afrontamiento adoptado. El CDPE-A, formato reducido actual, mantiene las mismas características iniciales de la estructura del CDPE ${ }^{(18)}$, y consta, además de las variables sociodemográficas, de 65 ítems que se responden mediante una escala de Likert con un rango de 1 a 4 , donde 1 es totalmente en desacuerdo y 4 totalmente de acuerdo. Este cuestionario incluye medidas de variables antecedentes propias del ámbito organizacional hospitalario como son la ambigüedad de rol, el contacto con el dolor y la muerte, la interacción conflictiva y la sobrecarga de trabajo (escala de antecedentes); medidas del propio síndrome de desgaste profesional habida cuenta las tres dimensiones clásicas propuestas por Maslach y Jackson en el inventario MBI, agotamiento emocional, 
despersonalización y realización personal, cambiando esta última por la análoga inversa "falta de realización personal" (escala del síndrome); y por último, recoge información sobre las diversas consecuencias del síndrome a nivel físico, psíquico, sociofamiliar y laboral (escala de consecuencias), así como de otras variables que pudieran intervenir en la génesis y desarrollo del desgaste profesional como la "personalidad resistente" y las estrategias de afrontamiento, a través de las dimensiones compromiso, reto, control (invertido), apoyo social, evitación y afrontamiento directo (escala de personalidad y afrontamiento)

Tratamiento de datos: El análisis de los datos fue estadístico, usando el programa informático SPSS 15 para Windows. Se utilizaron estadísticos descriptivos, porcentaje de medias, máximos, mínimos y desviaciones típicas para las variable de las escalas del CDPE-A; y estadística inferencial (con intervalos de confianza del 95\%) para averiguar la influencia de las variables sociodemográficas y laborales, a través de análisis bivariados, empleando la prueba t de Student para muestras independientes en variables dicotómicas, y ANOVA y contrastes Bonferroni en las variables politómicas; por último se ejecutaron análisis multivariados de varianzas (MANOVA).

\section{RESULTADOS}

En cuanto a las características de la muestra, por sexo supuso un $76,2 \%$ de mujeres frente un $23,8 \%$ de hombres, superando los 41 años el $83,3 \%$. Un porcentaje elevado tenía pareja estable $(78,5 \%)$ y con una distribución bastante similar entre el personal que no tenía hijos y el que tiene uno o dos. Siendo el $21,4 \%$ personal diplomado en enfermería, el 35,7\% personal técnico en cuidados auxiliares de enfermería y el $42,8 \%$ personal auxiliar de psiquiatría; la mayoría (80,9\%) tiene plaza fija y el $54,7 \%$ con más de 16 años de experiencia en el área de psiquiatría. En cuanto a jornada de trabajo, el $88,1 \%$ realiza jornada completa y el $83,3 \%$ turno rotatorio complejo. El tiempo de interacción (en este caso contacto directo y permanente) con la población psiquiátrica atendida es similar, al igual que el número de pacientes.

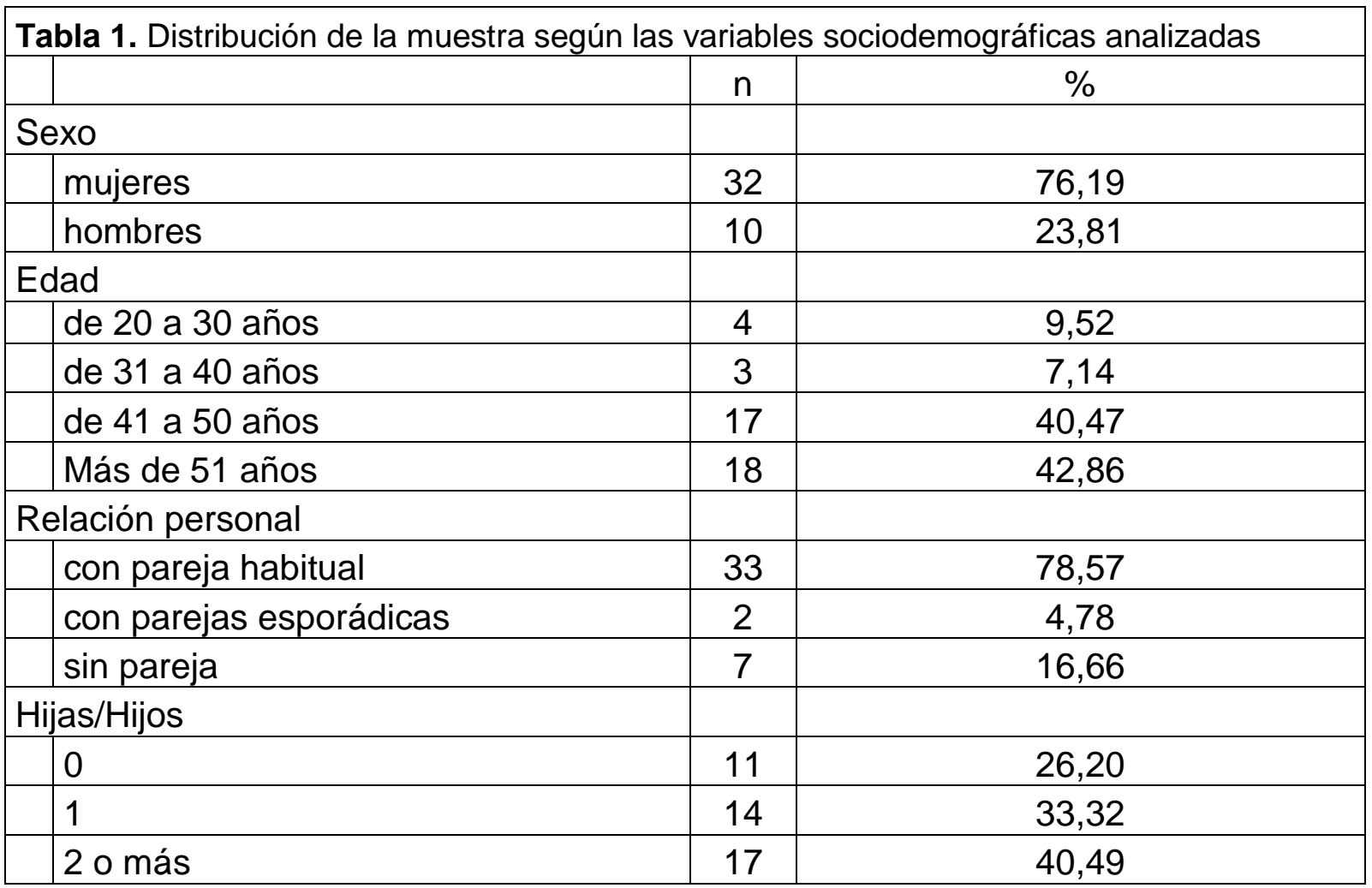




\begin{tabular}{|c|c|c|}
\hline & $\mathrm{n}$ & $\%$ \\
\hline \multicolumn{3}{|c|}{ Estudios/puesto de trabajo } \\
\hline DUE & 9 & 21,43 \\
\hline TCAE & 15 & 35,70 \\
\hline APS & 18 & 42,86 \\
\hline \multicolumn{3}{|l|}{ Situación laboral } \\
\hline Plaza fija & 34 & 80,95 \\
\hline Contrato & 6 & 14,29 \\
\hline Interinidad & 2 & 4,76 \\
\hline \multicolumn{3}{|c|}{ Antigüedad en el centro } \\
\hline De 0 a 5 años & 12 & 28,57 \\
\hline De 6 a 15 años & 7 & 16,61 \\
\hline De 16 a 30 años & 15 & 35,70 \\
\hline De 31 o más años & 8 & 19,05 \\
\hline \multicolumn{3}{|l|}{ Jornada laboral } \\
\hline completa & 37 & 88,10 \\
\hline $1 / 3$ de jornada & 3 & 7,14 \\
\hline $2 / 3$ de jornada & 2 & 4,76 \\
\hline \multicolumn{3}{|l|}{ Turno } \\
\hline mañana & 2 & 4,76 \\
\hline rotatorio simple & 5 & 11,90 \\
\hline rotatorio complejo & 35 & 83,30 \\
\hline
\end{tabular}

Los resultados para las cuatro escalas del CDPE-A obtenidos en esta muestra de 42 profesionales de enfermería fueron los siguientes:

En la escala del síndrome de desgaste profesional (ver Gráfica 1) se sitúan a niveles bajos un $60,0 \%$, a niveles medios un $37,5 \%$, y sólo un $2,5 \%$ a niveles altos, destacando los niveles medios alcanzados sólo para la dimensión cansancio $(57,2 \%)$.

Gráfica 1

\begin{tabular}{|c|c|c|c|c|c|}
\hline \multirow{12}{*}{$\begin{array}{l}\frac{\mathscr{c}}{\mathrm{U}} \\
\frac{\mathrm{d}}{\mathrm{J}} \\
\frac{\mathrm{d}}{4}\end{array}$} & \multicolumn{5}{|c|}{ Niveles Escala Síndrome Desgaste Profesional } \\
\hline & $70,00 \%$ & & & & \\
\hline & $60,00 \%$ & & & & \\
\hline & $50,00 \%$ & & & & \\
\hline & $40,00 \%$ & & & & \\
\hline & $30,00 \%$ & & & & \\
\hline & $20,00 \%$ & & & & \\
\hline & $10,00 \%$ & & & & \\
\hline & & $\begin{array}{l}\text { Escala del } \\
\text { Síndrome }\end{array}$ & Cansancio & $\begin{array}{c}\text { Despersona } \\
\text { lización }\end{array}$ & $\begin{array}{l}\text { Falta de } \\
\text { realización }\end{array}$ \\
\hline & - Baja & $60,00 \%$ & $23,80 \%$ & $63,40 \%$ & $63,40 \%$ \\
\hline & Media & $37,50 \%$ & $57,20 \%$ & $36,60 \%$ & $34,20 \%$ \\
\hline & Alta & $2,50 \%$ & $19,00 \%$ & $0 \%$ & $2,40 \%$ \\
\hline
\end{tabular}


En cuanto a la escala de antecedentes de desgaste profesional, propios del ámbito organizacional hospitalario e inherentes al trabajo e interpersonales, se encontraron niveles medios $(85,3 \%)$ para la escala en general, al igual que para las dimensiones ambigüedad de rol $(73,8 \%)$, interacción conflictiva $(64,3 \%)$ y sobrecarga $(58,5 \%)$, mientras que para la dimensión contacto con el dolor y la muerte los niveles alcanzados fueron altos $(66,7 \%)$

En la escala de consecuencias del síndrome también se aprecian niveles bajos en general $(53,7 \%)$, sobre todo en las consecuencias profesionales $(47,6 \%)$, y alcanzando unos niveles medios en las consecuencias psíquicas $(59,5 \%)$, las sociofamiliares $(50,0 \%)$ y las físicas $(61,0 \%)$.

La escala de personalidad incluye medidas para las tres dimensiones de personalidad resistente: compromiso, reto y control, y medidas para tres mecanismos de afrontamiento: apoyo social, evitación y afrontamiento directo. En la Gráfica 2 se pueden observar los niveles alcanzados para esta escala así como para cada una de sus dimensiones, siendo niveles medios para la escala general de personalidad y afrontamiento, y destacando los niveles altos en compromiso, reto, control, percepción de apoyo social y afrontamiento directo.

\section{Gráfica 2}

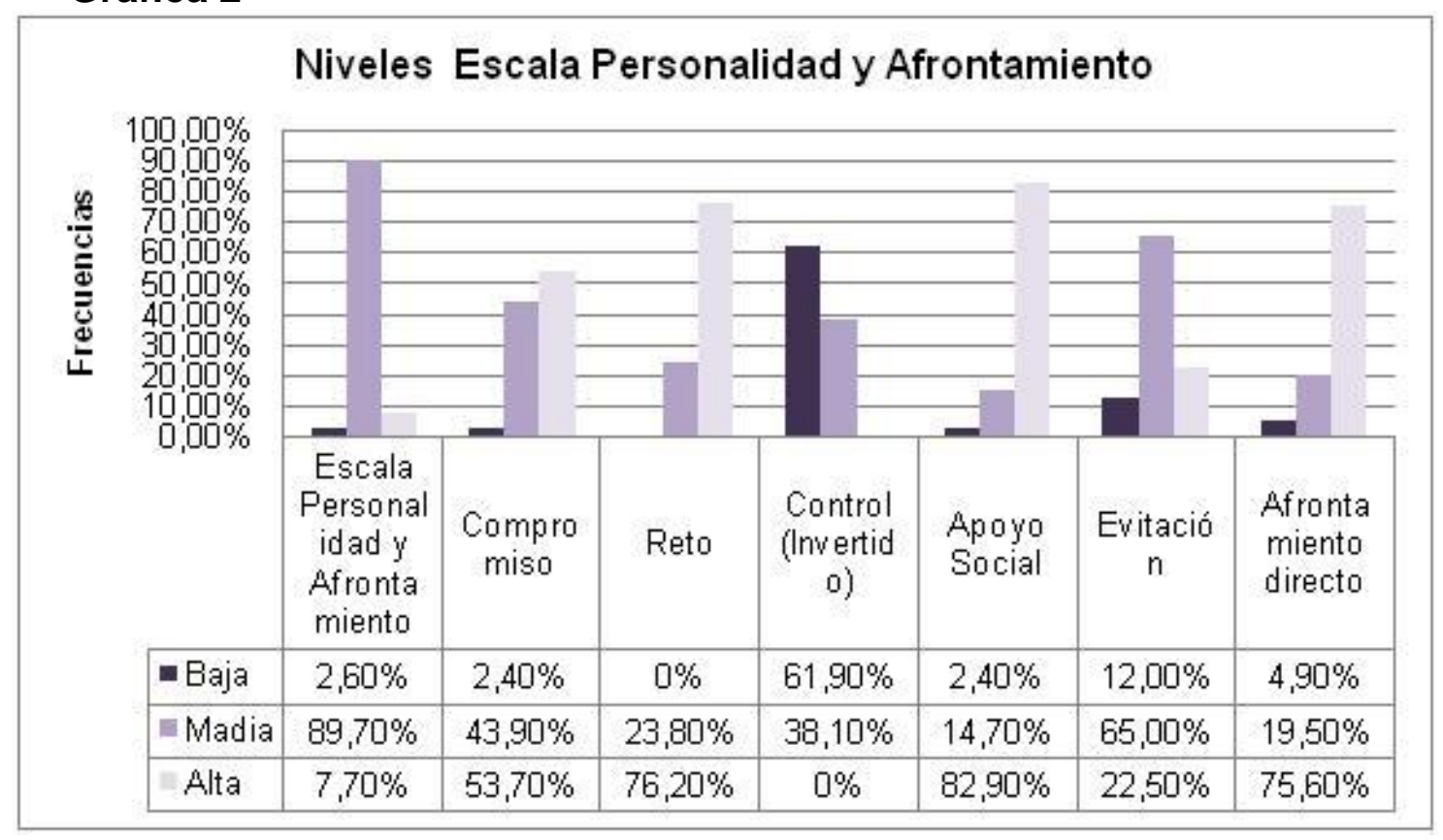

Tomando las variables sociodemográficas y laborales como variables independientes (ver tablas 1 y 2) y las puntuaciones obtenidas en las escalas de desgaste profesional como variables dependientes, no se encontraron efectos principales multivariados entre ellas, apreciándose sólo efectos significativos a nivel bivariado en función de la edad y el número de hijos. En cuanto a la edad, si bien a nivel escala de personalidad todos los grupos de edad alcanzan niveles medios, la variable que más contribuyó a la diferencia global entre el grupo de edad menor de 30 años y los grupos mayores de 41 años fue el apoyo social $(F=4,367 ; p=0,034)$; siendo el personal más joven el que presenta mayor percepción de apoyo social $(\mathrm{M}=3,92 ; s=0,16)$ y disminuyendo este a medida que aumenta la edad hasta los niveles medios alcanzados por el grupo de más de 51 años $(M=2,91 ; s=0,59)$. Si bien los resultados en las dimensiones de la escala del síndrome se encuentran en los mismos niveles y no se hallaron efectos 
principales, se observa menor desgaste general en el personal más joven aunque obtiene medias más altas en la dimensión cansancio. Igual sucede con la escala antecedentes donde el grupo de menos de 30 años alcanza niveles más altos en contacto con el dolor y la muerte, y también a nivel psicológico en la escala de consecuencias.

En relación al número de hijos aparecen efectos principales en la escala de antecedentes, encontrándose estos entre el personal que no tiene hijos y el que tiene sólo un hijo ( $F=4,152 ; p=0,041)$. Aún presentando niveles medios los dos grupos, el personal que no tiene hijos alcanza medias superiores en la escala de antecedentes, destacando en la dimensión sobrecarga donde llega a alcanzar niveles medios $(\mathrm{M}=2,52 ; s=0,74)$ frente a los niveles bajos obtenidos por el personal que tiene un sólo hijo $(M=1,96: s=0,29)$. Sin embargo los niveles en la escala del síndrome fueron bajos en estos dos grupos, pero alcanzando niveles medios el personal que tiene dos o más hijos, y sobre todo acusando más diferencia en la dimensión de cansancio.

\section{DISCUSIÓN}

La muestra analizada supone un $61 \%$ de la población a estudio por lo que se puede considerar representativa. Los análisis efectuados en este trabajo ponen de manifiesto que el desgaste profesional del personal de enfermería del Hospital de Rehabilitación Psiquiátrica $O$ Rebullón se encuentra dentro de un rango bajo y sólo un $2,5 \%$ alcanza niveles altos en la escala del síndrome. Si bien estos niveles de burnout fueron significativamente inferiores a algunos estudios nacionales ${ }^{(7,19)}$, también se hallaron resultados similares en otros ${ }^{(20)}$. Por otro lado, los resultados en la escala del síndrome concuerdan con los encontrados en la escala de consecuencias, donde aparece baja repercusión en el terreno laboral y moderada a nivel físico, psicológico y sociofamiliar.

Teniendo en cuenta los posibles predictores de este síndrome, relacionados con los antecedentes y factores organizacionales del trabajo, se han alcanzado niveles medios en ambigüedad de rol, interacción conflictiva y sobrecarga, y sólo destacan las puntuaciones altas en la dimensión de contacto con el dolor y la muerte, que suele ser uno de los estresores encontrados más frecuentemente en el personal de enfermería ${ }^{(21)}$.

Al analizar qué factores podrían estar modulando los resultados, en este estudio se confirmaría que las variables sociodemográficas clásicamente estudiadas tienen escasa relación con la aparición del burnout, pues no llegaron a encontrarse efectos significativos en la escala del síndrome, y sólo aparecen efectos significativos en función de la variable edad en la escala de personalidad y de la variable número de hijos en la escala de antecedentes. En cuanto a la edad, la literatura discrepa al asociar los años de experiencia con el desarrollo del síndrome, y en ocasiones muestra una relación inversa entre este y la edad, bien por el aprendizaje de estrategias de afrontamiento eficaces o por un sesgo de supervivencia, suponiendo que el personal más quemado es el que mayoritariamente abandona el trabajo (22). Aquí nos encontramos que a pesar de no hallar efectos principales en las dimensiones del síndrome, y encontrándose a niveles iguales todos los grupos de edad establecidos, se observa menos desgaste general en el personal menor de 30 años, que sin embargo presenta más cansancio emocional y más vulnerabilidad al contacto con el sufrimiento, repercutiendo también a nivel psicológico. 
Las diferencias sólo son significativas en la dimensión de apoyo social de la escala de personalidad, destacando los niveles máximos alcanzados en el grupo de edad menor de 30 años y bajando con los años hasta niveles medios a partir de los 51 años. Igual sucede con la variable número de hijos, que si bien en algunas publicaciones se observa mayor vulnerabilidad al agotamiento emocional en sujetos que no tienen hijos ${ }^{(23)}$, alcanzando cotas máximas de desgaste profesional ${ }^{(12)}$, que lleva a considerar que la existencia de hijos puede contribuir a la resistencia del desarrollo del síndrome por el aumento de capacidad para afrontar problemas y conflictos emocionales ${ }^{(24)}$; sin embargo, en nuestro caso, y aunque no aparecen efectos significativos en ninguna de las dimensiones de la escala del síndrome, encontramos que con más de un hijo se invierten los resultados, es decir, aumenta el desgaste profesional sobre todo en cansancio emocional, y solo aparecen efectos principales en la escala de antecedentes entre las personas que tienen un solo hijo y ninguno.

En cuanto a la falta de efectos principales significativos bivariados del resto de las variables estudiadas como fueron sexo, relaciones personales, categoría profesional, turno, antigüedad, situación y jornada laboral, sobre las dimensiones de la escala de desgaste profesional, habrá que tener en cuenta las limitaciones de este estudio, es decir, el tamaño muestral y la distribución asimétrica entre los distintos subgrupos de algunas variables, que podrían estar condicionando el establecimiento de relaciones estadísticamente significativas

Por otro lado, teniendo en cuenta los resultados de esta investigación, cabría preguntarse si la personalidad resistente o fortaleza, considerada como factor protector frente el burnout, también estaría modulando la génesis y el desarrollo del desgaste profesional en estas unidades de rehabilitación psiquiátrica. A la luz de los resultados hallados, sobre todo en las dimensiones de compromiso y reto, podría interpretarse que la personalidad resistente sería la variable que más influencia ejerce, en este caso bloqueando el desarrollo del desgaste profesional, así como los fuertes mecanismos de afrontamiento que presenta esta muestra de personal de enfermería y recogidos como niveles altos de percepción de apoyo social y afrontamiento directo, que son considerados como factores positivos en la prevención del burnout ${ }^{(25)}$.

También, al igual que otros estudios en personal de enfermería psiquiátrica ${ }^{(11)}$, se encontraron niveles altos de percepción de apoyo social frente a niveles bajos y moderados de desgaste profesional, lo que corroboraría que la percepción de cohesión y compañerismo ejerce una acción protectora ante el desgaste, pues a mayor apoyo y reconocimiento profesional recibido en el trabajo, ya sea de compañeros 0 de superiores, es menor el agotamiento emocional, la despersonalización y la baja realización profesional ${ }^{(26)}$.

Por último, dado que este estudio también sugiere que algunas variables de personalidad pudieran predecir el proceso de desgaste, pues los niveles alcanzados en las dimensiones de personalidad se relacionan inversamente con los niveles de desgaste profesional, que resultó ser bajo en general aunque alto en cansancio emocional $(23,8 \%)$, sobre todo en el personal con menos experiencia laboral en esta área, convendría plantearse tomar medidas adecuadas para evitar el desarrollo del síndrome y considerar alguna intervención enfocada al incremento de habilidades de afrontamiento de estrés, de autocontrol y de comunicación asertiva, dirigidas a potenciar al desarrollo de una personalidad resistente que permita optimizar el manejo 
de las situaciones de estrés inherentes a la profesión de enfermería en el área de psiquiatría y así poder realizar una prevención temprana. Al igual, sería interesante contar con futuros trabajos que permitan identificar los factores organizacionales y personales que puedan estar impactando en la profesión de enfermería de otros servicios de psiquiatría que no se dediquen a la rehabilitación, teniendo en cuenta, como ya se ha comentado, que el manejo de pacientes psiquiátricos resulta altamente estresante si se carece de ciertas habilidades.

\section{CONCLUSIONES}

Este estudio pone de manifiesto que a pesar de ser el personal de enfermería una población susceptible de padecer desgaste profesional provocado por el agotamiento derivado de factores tanto organizacionales e interpersonales como de las características especificas de interacción, directa y permanente del área de rehabilitación psiquiátrica, pueden ser muchos otros los factores que influyen en este proceso a la vista de los niveles bajos del síndrome de burnout hallados en la población investigada, donde sólo destacan los niveles altos alcanzados en la dimensión contacto con el dolor y la muerte de la escala de antecedentes, y sin apenas repercusión en la escala de consecuencias. También se observó poca relación con los factores sociodemográficos y laborales, encontrando sólo diferencias significativas a nivel bivariado en función de la edad y número de hijos. Por último, y dada la incidencia de niveles bajos en burnout y de niveles medio-altos en todas las dimensiones de fortaleza y mecanismos de afrontamiento como fueron compromiso, reto, control, percepción de apoyo social, evitación y afrontamiento directo en esta población, lo que sugiere que podrían contribuir al bloqueo del desgaste profesional, habrá que tener en consideración las variables de personalidad a la hora de estudiar el proceso de desgaste profesional.

\section{Agradecimientos}

Al personal de enfermería del Servicio de Rehabilitación Psiquiátrica de O Rebullón; a los profesores José Manuel Dos Santos (Universidad Fernando Pessoa de Oporto), Jacinto González Oya (Escuela Universitaria de Enfermería de Vigo) y Bernardo Moreno Jimenez (Universidad Autónoma de Madrid); y a Manoli Fontanillo Fontanillo y Angel Salgado Barreiro de la Unidad de Apoyo a la Investigación del Chuvi. Po último a Maria Teresa Troncoso Lago por la traducción al inglés.

\section{BIBLIOGRAFÍA}

(1) Grossoni, M. (2007). Salva tu vida. Madrid. Ed. LID Acción Empresarial.

(2) Gil-Monte, P. (2011) El síndrome de quemarse por el trabajo (burnout). Madrid. Ediciones Pirámide.

(3) Moreno B, Rodríguez RB, Álvarez AM, Caballero TM (1997). La evaluación del burnout. Problemas y alternativas. El CBB como evaluación de los elementos del proceso. Revista de Psicología del Trabajo y de las Organizaciones, 13, 2, pp.185-207 (4) Salanova M y Llorens S (2008) Estado actual y retos futuros en el estudio del burnout. Madrid. Papeles del Psicólogo. Vol 29 (1), pp. 59-67. (5) Gallegos M, Parra ML, Castillo R, Murillo E (2009) Burn-out syndrome and PsychoSocial factors of nurses in the care of Oncologic patient. México. Revista Conamed. 
Vol 14, pp 28-33. (acceso en junio 2012). Disponible en http://www.conamed.gob.mx/publicaciones/pdf/REV_ENERO-MARZO_2009.pdf

(6) García AS, Espinosa EC, Jiménez MC, González MB, Soto PM, Padro AM (2012). Síndrome de burnout en el personal sanitario de una residencia asistida de mayores. Revista de enfermería ROL. Vol 35, no 10, pp16-22

(7) Garrosa E, Moreno B, Liang Y, González JL (2008) The relationship between sociodemographic variables, job stressors, burnout, and hardy personality in nurses: An exploratory study. Internacional Journal of Nursing Studies. Vol 45, núm. 3, pp. 418-427

(8) Palmer Y, Principe R, Searcy R y Compean B(2007) Prevalencia del síndrome de burnout en el per.sonal de enfermería de 2 hospitales mexicanos. México. Enfermería Clínica Vol 17, núm 5, pp. 256-60.

(9) Gómez MC, Álamo MC, Amador M, Ceacero F, Mayor A, Muñoz A, Izquierdo $M(2009)$ Estudio de seguimiento del desgaste profesional en relación con factores organizativos en el personal de enfermería de medicina interna. Medicina y Seguridad del Trabajo Vol 55 (215), pp. 52-62. (acceso en junio 2012) Disponible en http://scielo.isciii.es/scielo.php?pid=S0465546X2009000200005\&script=sci_arttext

(10) Caballero M.A, Bermejo F, Nieto R, Caballero F (2001) Prevalencia y factores asociados al burnout en un área de salud. Madrid. Atención Primaria Vol. 27, núm. 5. Pp. 313-317.

(11) Aldali E, Priami M, Evagelou H, Mougia V, Ifanti M, Alevizopoulos G (2003) Síndrome del Quemado en el personal de enfermería psiquiátrica de hospitales griegos. The European Journal of Psychiatria. Vol 17, núm 3, pp. 161-170. (acceso en junio 2012) Disponible en http://scielo.isciii.es/scielo.php?script=sci_arttext\&pid=S1579-699X2003000300004

(12) Figueiredo H, Grau E, Gil R, García JA (2012) Síndrome de quemarse por el trabajo y satisfacción laboral en profesionales de enfermería. Psicothema. Vol. 24, núm. 2, pp. 271-276.

(13) Moreno B, González JL, Garrosa E (2002) Variables sociodemográficas en el proceso de desgaste profesional de enfermería. Rol de Enfermería. Vol 25, núm 11, pp 18-26.

(14) Moreno B, Morante ME, Rodríguez R, Rodríguez A (2008) Resistencia y vulnerabilidad ante el trauma: el efecto moderador de las variables de personalidad. Psicothema. Vol 20, núm. 1, pp 124-130.

(15) Ríos MI, Sánchez J, Godoy C (2010) Personalidad resistente, autoeficacia y estado general de salud en profesionales de enfermería de cuidados intensivos y urgencias. Psicothema. Vol 22, núm. 4, pp. 600-605.

(16) INFOCOP, Redacción (2009). Salud Mental y absentismo laboral: un binomio por resolver. Madrid. Revista INFOCOP, núm. 41, pp 4-8.

(17) Tomás J, Maynegre M, Pérez M, Alsina M, Quinta R, Granell S (2010) Síndrome de burnout y riesgo suicida en enfermeras de atención primaria. Enfermería Clínica. Vol 20, núm 03, pp. 173-8

(18) Moreno, B. Garrosa, E. y González, J.L. (2000) El Desgaste profesional de enfermería. Desarrollo y validación factorial del CDPE. Archivos Prevención de Riesgos Laborales, 3, 1, pp. 18-28.

(19) Puialto M.J. Antolín R. y Moure L. (2006) Prevalencia del síndrome del quemad@ y estudio de factores relacionados en I@s enfermer@s del CHUVI. Rev. Enfermería Global, núm. 8. Ed. Universidad de Murcia. (acceso en marzo 2012) Disponible en http://revistas.um.es/eglobal( /article/view/320/300)

(20) Ríos MI, Martínez F, Sabuco E, Lozano E, Mateo G (2012). Quiero cambiar de servicio ¿estoy quemado? Revista de enfermería ROL vol 35, no 5, pp 56-61 
(21) López MP, Torrejón G, Martín A, Martín A, Martín J, Pleite F, Torres MA (2011) Estrés y satisfacción laboral de las enfermeras de hospitales toledanos. Revista Metas de Enfermería. Vol 14, núm. 8, pp. 8-14

(22) Grau A, Suñer R, García MM (2005) Desgaste profesional en el personal sanitario y su relación con los factores personales y ambientales. Gaceta Sanitaria. Vol 19, núm. 6, pp 463-70

(23) Ríos MI, Peñalver F, Godoy C (2008) Burnout y salud percibida en profesionales de enfermería de Cuidados Intensivos. Murcia. Enfermería Intensiva. Vol 19, núm 4, pp. 169-78.

(24) Díaz C, Pérez T. (2007) Prevalencia do Bournout en enfermeros/as de atención primaria. Lugo. Enfermeir@s. Revista do Colexio Oficial de Enfermería de Lugo. Núm. 10, pp.44-48.

(25) Garrosa E, Rainho C, Moreno-Jiménez B, Monteiro MJ (2010) The relationship between job stressors, hardy personality, coping resources and burnout in a sample of nurses: A correlational study at two time points Internacional Journal of Nursing Studies. Vol 45, núm. 3, pp. 418-427

(26) Avendaño C, Bustos P, Espinoza P, García F, Pierart T (2009) Burnout y apoyo social en personal del servicio de psiquiatría de un hospital público. Chile. Ciencia y Enfermería. Vol. XV (2), pp. 55-68. 